\title{
Prescribed Performance Tracking Control for Hypersonic Flight Vehicles with Model Uncertainties
}

\author{
Siyuan Zhao $\mathbb{D}^{1}{ }^{1}$ Xiaobing $\mathrm{Li}^{2}{ }^{2}$ Xiangwei Bu ${ }^{\circ},{ }^{2}$ and Dongyang Zhang ${ }^{2}$ \\ ${ }^{1}$ Graduate College, Air Force Engineering University, Xi'an 710051, China \\ ${ }^{2}$ Air and Missile Defense College, Air Force Engineering University, Xi'an 710051, China \\ Correspondence should be addressed to Siyuan Zhao; 1098547574@qq.com
}

Received 25 June 2019; Accepted 6 September 2019; Published 13 October 2019

Academic Editor: Rosario Pecora

Copyright ( 2019 Siyuan Zhao et al. This is an open access article distributed under the Creative Commons Attribution License, which permits unrestricted use, distribution, and reproduction in any medium, provided the original work is properly cited.

\begin{abstract}
This paper proposes a novel prescribed performance tracking control for a hypersonic flight vehicle (HFV) with model uncertainties. Firstly, a HFV longitudinal motion model is decomposed into a velocity subsystem and an altitude subsystem. Meanwhile, considering the uncertainties of the model, the velocity subsystem and altitude subsystem are directly expressed as the forms with unknown nonaffine functions. Secondly, a novel performance function without initial error is proposed for limiting the tracking error into a prescribed range. Then, for the altitude subsystem, the control objective is changed by model transformation and the prescribed performance backstepping controller is designed. For the velocity subsystem, a prescribed performance proportional-integral controller is proposed which has better engineering practicability. The designed controller is not only simple in form but also has few calculating parameters. Finally, the simulation results show that the proposed controller has good practicability.
\end{abstract}

\section{Introduction}

Near space usually refers to the $20 \sim 100 \mathrm{~km}$ airspace from the horizontal plane [1]. This airspace is located between the traditional concept of "aviation" and "aerospace," across the stratosphere and ionosphere, which is rarely involved in the past aircraft airspace. Thus, the development prospects are very broad $[2,3]$. A flight vehicle flying in near space and with hypersonic speed is called a hypersonic flight vehicle (HFV). HFV is considered as a key step towards providing a promising and affordable technology to meet commercial and military goals [4]. Compared with traditional aircraft, HFV has stronger nonlinear and coupling characteristics, more serious elastic vibration, and more strict control constraints. The complex dynamic characteristics of HFV will undoubtedly bring great challenges to the design of its controller, which makes the HFV control problem a hot research topic [5-7].

The modeling and control design of HFV are mainly carried out on its longitudinal motion model. On one hand, its longitudinal motion model for flight control is complicated enough; on the other hand, considering the scramjet's sensitivity to flight attitude and fuel saving, lateral maneuver should be avoided as much as possible during actual cruise flight [8]. Due to the difficulty to establish a precise motion model for HFV, Ref. [9] investigates a novel model-free controller for the HFV longitudinal motion model. Since the HFV model has fast time variability, strong coupling, and highly nonlinear and uncertain parameters, a sliding-mode decoupling attitude controller based on parametric commands is proposed which takes these features into account [10]. In order to facilitate the design of the controller, the input and output linearized high-order model of HFV is transformed into a multivariable second-order system model by introducing auxiliary error variables. Meanwhile, in order to ensure the actual finite-time stability of the sliding-mode manifold, an adaptive fast nonsingular terminal slidingmode controller is designed for HFV with an unknown upper limit of disturbance [11].

The basic idea of a backstepping control design is to decompose the complex nonlinear system into subsystems with no more than orders of the system, and then design a 


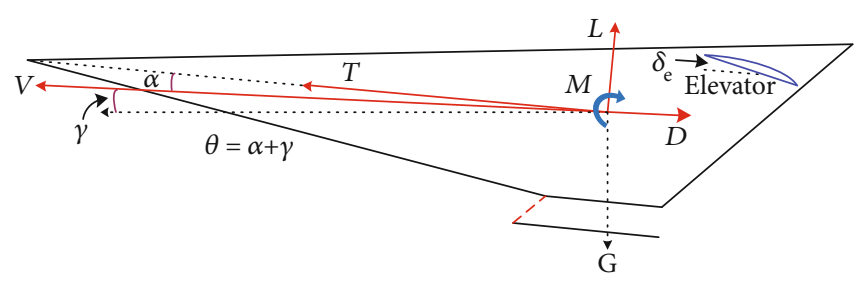

FIGURE 1: Geometry and force map of HFV model.

Lyapunov function and an intermediate virtual control quantity for each subsystem, then push it to the whole system, and complete the design of the whole control law by integrating them [12]. Backstepping control is suitable for uncertain nonlinear systems with state linearization or strict parameter feedback, especially in the field of aeronautics and astronautics [13]. At the same time, intelligent control has good performance in dealing with model uncertain control problems [14-16]. Therefore, many studies have combined the idea of an intelligent control and backstepping control method to solve the HFV control problem. Ref. [17] discusses the design of HFV adaptive fuzzy backstepping tracking control with actuator constraints and applies the fuzzy logic system to approximate analysis of the total uncertainty of the fuzzy fractal method model. Based on the control-oriented model of HFV, an adaptive backstepping controller and a dynamic inverse controller are designed for the altitude subsystem and velocity subsystem, respectively, in Ref. [18]. Meanwhile, in order to avoid the complexity problems caused by the repeated derivation of virtual control variables by traditional synchronization control, the dynamic surface control technique and synchronization control method are involved, and a novel second-order sliding-mode-based integral filter is used to replace the traditional first-order filter. In order to compensate for the dead zone effect of HFV and reduce the computation load, an improved dead zone smooth inverse is proposed, on which basis an input nonlinear precompensator is designed to deal with input saturation and dead zone nonlinearity [19].

The existing control methods pay less attention to the overall control performance of HFV, but too much attention is paid to the steady progress of the control system $[20,21]$. The prescribed performance control method requires that the system can meet the expected dynamic performance and steady performance at the same time, so as to improve the integrity of the system for the control goal, which has extremely important theoretical and practical significance [22]. Different from the traditional nonaffine model that requires nonaffine functions to be differentiable, Ref. [23] uses the semidecomposed nonaffine model to design an improved performance controller with a simple structure and low computational complexity on the basis of the backextrapolation technique.

Consistent with a quadrotor unmanned aerial vehicle system proposed in Ref. [24], the actual control model of HFV is also uncertain. Few examples of previous studies have applied HFV's uncertain nonaffine model in the research of prescribed performance tracking control. In order to improve the overall control performance of HFV with an unknown model, a pre- scribed performance tracking control is designed in this paper. Firstly, the HFV longitudinal motion model is decomposed into a velocity subsystem and an altitude subsystem. At the same time, considering the uncertainty of the model, the velocity subsystem and the altitude subsystem are directly expressed as the forms with unknown nonaffine functions. Secondly, a new performance function without initial error is proposed to limit the tracking error within the specified range. Then, the control target of the altitude subsystem is changed by model transformation, and the corresponding backstepping controller is designed. Unlike previous studies, the backstepping controller designed in this paper reduces the steps and computational load. A prescribed performance Proportional-Integral (PI) controller is proposed for the velocity subsystem. The controller is simple in form and has few calculating parameters. Simulation results show that the controller has good practicability. The main contributions of this paper are summarized as follows:

(1) The control method designed in this paper is based on the uncertain nonaffine models of HFV, which are closer to the actual flight situation than previous studies

(2) The novel prescribed performance function is introduced to ensure transient performance and steadystate accuracy without initial error

(3) The uncertainties in the HFV flight control are realized by using the backstepping control method to meet the requirements of matching conditions. There are less steps in the backstepping design and low computational load. Meanwhile, a PI controller, which is more commonly used in engineering practice, is applied in the velocity subsystem

(4) Finally, the simulation results are compared with several methods proposed in existing references under different conditions

The rest of this paper is organized as follows. The HFV model description and transformation are described in Section 2, and the control laws are addressed in Section 3. In Section 4, the simulation results are presented. Finally, Section 5 provides the conclusion of this paper.

\section{Model Description and Transformation}

2.1. Model Description. As is shown to us, the longitudinal models of the controller design for HFV are usually 
considered as follows. The winged-cone rigid-body model of NASA is mostly used in the early Ref. [25]. The disadvantage of this model is that the elastic effect of HFV during flight is not taken into account. In order to consider the influence of the elastic state, the integrated analytical model [26] and the improved analytical model [27, 28] are proposed. On the basis of these two models, Parker, a researcher in the United States Air Force Research Office, established the HFV control-oriented parameter fitting model through the Hooke law and the Lagrange theorem [7]. In this study, we consider the longitudinal model of HFV developed by Parker. The geometry and force map of the HFV models are shown in Figure 1.

$$
\begin{aligned}
\dot{V} & =\frac{T \cos (\theta-\gamma)}{m}-\frac{D}{m-g \sin \gamma}, \\
\dot{h} & =V \sin \gamma, \\
\dot{\gamma} & =\frac{L}{m V}+\frac{T \sin (\theta-\gamma)}{m V}-g \cos \gamma, \\
\dot{\theta} & =Q, \\
\dot{Q} & =\frac{\left(M+\tilde{\psi}_{1} \ddot{\eta}_{1}+\tilde{\psi}_{2} \ddot{\eta}_{2}\right)}{I_{\mathrm{yy}}}, \\
k_{1} \ddot{\eta}_{1} & =-2 \zeta_{1} \omega_{1} \dot{\eta}_{1}-\omega_{1}^{2} \eta_{1}+N_{1}-\frac{\ddot{\psi}_{1} M}{I_{\mathrm{yy}}}-\frac{\tilde{\psi}_{1} \tilde{\psi}_{2} \ddot{\eta}_{2}}{I_{\mathrm{yy}}}, \\
k_{2} \ddot{\eta}_{2} & =-2 \zeta_{2} \omega_{2} \dot{\eta}_{2}-\omega_{2}^{2} \eta_{2}+N_{2}-\frac{\tilde{\psi}_{2} M}{I_{y y}}-\frac{\tilde{\psi}_{2} \tilde{\psi}_{1} \ddot{\eta}_{1}}{I_{y y}},
\end{aligned}
$$

where five rigid-body states $V, h, \gamma, \theta$, and $Q$ represent velocity, altitude, flight-path angle, pitch angle, and pitch rate, respectively. $\eta_{1}$ and $\eta_{2}$ are the elastic states. $T, D, L, M$, $N_{1}$, and $N_{2}$ mean the thrust force, the drag force, the lift force, the pitching moment, the first generalized force, and the second generalized force which are defined, respectively, as follows [7]:

$$
\begin{aligned}
T \approx & \beta_{1}(h, \bar{q}) \Phi \alpha^{3}+\beta_{2}(h, \bar{q}) \alpha^{3}+\beta_{3}(h, \bar{q}) \Phi \alpha^{2}+\beta_{4}(h, \bar{q}) \alpha^{2} \\
& +\beta_{5}(h, \bar{q}) \Phi \alpha+\beta_{6}(h, \bar{q}) \alpha+\beta_{7}(h, \bar{q}) \Phi+\beta_{8}(h, \bar{q}) \\
D \approx & \bar{q} S C_{D}^{\alpha^{2}} \alpha^{2}+\bar{q} S C_{D}^{\alpha} \alpha+\bar{q} S C_{D}^{\delta_{e}^{2}} \delta_{e}^{2}+\bar{q} S C_{D}^{\delta_{e}} \delta_{e}+\bar{q} S C_{D}^{0}, \\
M \approx & z_{T} T+\bar{q} S c C_{M, \alpha}^{\alpha^{2}} \alpha^{2}+\bar{q} S c C_{M, \alpha}^{\alpha} \alpha+\bar{q} S \bar{c} C_{M, \alpha}^{0}+\bar{q} S c c_{e} \delta_{e} \\
L \approx & \bar{q} S C_{L}^{\alpha} \alpha+\bar{q} S C_{L}^{\delta_{e}} \delta_{e}+\bar{q} S C_{L}^{0}, \\
N_{1}= & N_{1}^{\alpha^{2}} \alpha^{2}+N_{1}^{\alpha} \alpha+N_{1}^{0}, \\
N_{2}= & N_{2}^{\alpha^{2}} \alpha^{2}+N_{2}^{\alpha} \alpha+N_{2}^{\delta_{e}} \delta_{e}+N_{2}^{0}, \\
\bar{q}= & \frac{\bar{\rho} V^{2}}{2}, \bar{\rho}=\bar{\rho}_{0} \exp \left[\frac{-\left(h-h_{0}\right)}{h_{s}}\right]
\end{aligned}
$$

where the control inputs $\Phi$ and $\delta_{e}$ stand for fuel equivalence ratio and elevator angular deflection, and they occur implicitly in (1), (2), (3), (4), (5), (6), and (7). Should the readers want to know more about parameters and aerodynamic parameters in the above equations, they could refer to Ref. [7].

Usually, only the rigid-body states $V, h, \gamma, \theta$ and $Q$ of HFV are measurable which can be utilized in the design of control laws. Also, the elastic states $\eta_{1}$ and $\eta_{2}$ are considered as external disturbances when the controller is designed because they are not measurable.

Definition $1[29,30]$. A continuous function $\rho(t)$ that satisfies the following two conditions simultaneously is called a performance function:

(1) $\rho(t)$ is continuously differentiable, bounded, strictly positive, and has a decreasing function of time

(2) $\lim _{t \rightarrow \infty} \rho(t)=\rho_{\infty}>0$.

According to Definition 1, this paper chooses the following function as the performance function:

$$
\rho(t)=\operatorname{csch}\left(l t+\rho_{0}\right)+\rho_{\infty},
$$

where $l, \rho_{0}$, and $\rho_{\infty}$ are positive parameters. $l$ is relative to the convergence rate of the tracking error. $\rho_{0}$ is relative to the boundedness of the tracking error. $\rho(0)=\operatorname{csch}\left(\rho_{0}\right)+\rho_{\infty}$ represents the maximum overshoot of the tracking error. The convergence interval of the tracking error $e(t)$ is defined as

$$
-\rho(t)<e(t)<\rho(t)
$$

Obviously, it is impossible to design the controller directly from inequality (10). In this paper, we introduce an error transformation function as follows:

$$
T(\varepsilon)=\frac{e^{\varepsilon(t)}-e^{-\varepsilon(t)}}{e^{\varepsilon(t)}+e^{-\varepsilon(t)}},
$$

where $\varepsilon(t)$ is the transform error.

2.2. Model Transformation. According to the timescale principle in [31], velocity has slower dynamics compared with altitude angles. Thus, the HFV motion model can be decomposed into a velocity subsystem and an altitude subsystem.

A velocity subsystem can be represented as follows:

$$
\dot{V}=f_{0}(V, \Phi)
$$

where $f_{0}(V, \Phi)$ is a continuous unknown differentiable nonaffine function.

The altitude tracking error $\tilde{h}$ is defined as

$$
\tilde{h}=h-h_{\text {ref }} .
$$


The reference command of $\gamma$ is designed as

$$
\gamma_{d}=\arcsin \left(\frac{-k_{h} \varepsilon_{h}(t)+\dot{h}_{r e f}}{V}\right)
$$

where $k_{h}>0$ is the design parameter. Meanwhile, $\varepsilon_{h}(t)=\ln$ $\left(\left(\tilde{h} / \rho_{h}(t)+1\right) /\left(1-\tilde{h} / \rho_{h}(t)\right)\right)$ is the altitude transform error and $\rho_{h}(t)=\operatorname{csch}\left(l_{h} t+\rho_{h 0}\right)+\rho_{h \infty}$.

If $\gamma \rightarrow \gamma_{d}$, the corresponding dynamics for $\varepsilon_{h}(t)$ is derived as

$$
\dot{\varepsilon}_{h}(t)+k_{h} \varepsilon_{h}(t)=0 .
$$

Obviously, if $\varepsilon_{h}(t)$ is bounded, the transient performance and steady-state performance of $\tilde{h}$ are both guaranteed $[32,33]$. Thus, the control objective of the altitude subsystem is changed into $\gamma \rightarrow \gamma_{d}$.

In this way, the subsequent design goal is to let $\gamma \rightarrow \gamma_{d}$. Then, the altitude subsystem is changed into

$$
\left\{\begin{array}{l}
\dot{x}_{1}=f_{1}\left(x_{1}, x_{2}\right)+x_{2} \\
\dot{x}_{2}=f_{2}(\mathbf{x})+x_{3} \\
\dot{x}_{3}=f_{3}\left(\mathbf{x}, \delta_{\mathrm{e}}\right)+\delta_{\mathrm{e}}
\end{array}\right.
$$

where $f_{1}\left(x_{1}, x_{2}\right), f_{2}(\mathbf{x})$, and $f_{3}\left(\mathbf{x}, \delta_{e}\right)$ are continuous unknown differentiable nonaffine functions, $\mathbf{x}=\left[x_{1}, x_{2}, x_{3}\right]^{T}$, where $x_{1}=\gamma, x_{2}=\theta$, and $x_{3}=Q$.

According to the prescribed performance control method, we consider the following change of coordinate:

$$
\tilde{\gamma}=x_{1}-\gamma_{d}
$$

where $\tilde{\gamma}$ is the error flight-path angle. The time derivative of (17) is as follows:

$$
\dot{\tilde{\gamma}}=f_{1}\left(x_{1}, x_{2}\right)+x_{2}-\dot{\gamma}_{d}
$$

Based on $e(t)=\rho(t) T(\varepsilon(t))$, the time derivative of the transform error $\varepsilon_{1}(t)$ of the flight-path angle is as follows:

$$
\dot{\varepsilon}_{1}(t)=\frac{\tilde{\gamma}-\dot{\rho}_{1}(t) T\left(\varepsilon_{1}(t)\right)}{\rho_{1}(t) \partial T\left(\varepsilon_{1}(t)\right) / \partial \varepsilon_{1}(t)} .
$$

Meanwhile, $\varepsilon_{1}(t)=\ln \left(\left(\tilde{\gamma} / \rho_{1}(t)+1\right) /\left(1-\tilde{\gamma} / \rho_{1}(t)\right)\right)$ and $\rho_{1}(t)=\operatorname{csch}\left(l_{1} t+\rho_{10}\right)+\rho_{1 \infty}$. Let $\Gamma=-\left(\dot{\rho}_{1}(t) T\left(\varepsilon_{1}(t)\right) /\left(\rho_{1}(t)\right.\right.$ $\left.\left.\partial T\left(\varepsilon_{1}(t)\right) / \partial \varepsilon_{1}(t)\right)\right)$ and $\Lambda=1 /\left(\rho_{1}(t) \partial T\left(\varepsilon_{1}(t)\right) / \partial \varepsilon_{1}(t)\right)$, then the above altitude subsystem is modified as

$$
\left\{\begin{array}{l}
\dot{\varepsilon}_{1}(t)=\Gamma+\Lambda\left(f_{1}\left(x_{1}, x_{2}\right)+x_{2}-\dot{\gamma}_{d}\right), \\
\dot{x}_{2}=f_{2}(\mathbf{x})+x_{3}, \\
\dot{x}_{3}=f_{3}\left(\mathbf{x}, \delta_{e}\right)+\delta_{e} .
\end{array}\right.
$$

\section{Controller Design}

The control objective is to devise prescribed performance tracking control laws $\Phi$ and $\delta_{e}$, such that $V$ and $h$ can accurately track their reference inputs $V_{\text {ref }}$ and $h_{\text {ref }}$. Meanwhile, the control laws need to guarantee tracking errors with a prescribed transient. To achieve the control goal, the following lemmas and assumption need to be considered.

Lemma 1 [34]. The unknown nonlinear function $f_{1}, f_{2}$, and $f_{3}$ satisfy the following inequality:

$$
\left|f_{i}\left(\bar{x}_{i}\right)\right| \leq\left(\left|x_{1}\right|+\cdots+\left|x_{i}\right|\right) \Upsilon_{i}\left(x_{1}, \cdots, x_{i}\right), \quad i=1, \cdots, 3,
$$

where $\Upsilon_{i}\left(x_{1}, \cdots, x_{i}\right)$ are the known $C^{1}$ functions.

Assumption $1[35,36]$. For the unknown velocity subsystem (12) and altitude subsystem (16), the desired trajectory and its derivative are bounded.

Lemma 2 [37, 38]. Supposing the nonnegative definite function $g(t)$ satisfies

$$
\frac{d g(t)}{d t}=-g_{1}[g(t)]^{g_{2}}
$$

where $g_{1}>0$ and $0<g_{2}<1$ are design constants. Then, by solving the above differential equation, we can get

$$
g(t)=\left\{\begin{array}{l}
{\left[(g(0))^{1-g_{2}}-g_{1}\left(1-g_{2}\right) t\right]^{1 / 1-g_{2}}, \quad t \in\left[0, t_{0}\right)} \\
0, \quad t \in\left[t_{0},+\infty\right)
\end{array}\right.
$$

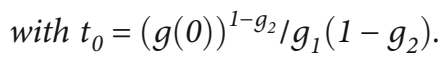

3.1. Altitude Control Design. By considering equations (10), (11), and (17), there are $\left|x_{1}\right| \leq \rho_{1}(t)\left|\varepsilon_{1}(t)\right|+\left|\gamma_{d}\right|$ and $\left|x_{i}\right| \leq \mid$ $e_{i}|+| \alpha_{i-1} \mid$ with $e_{i}=x_{i}-\alpha_{i-1}$, where $\alpha_{i-1}$ is the virtual controller.

Step 1. It follows that from Lemma 1 and Young's inequality, there is

$$
\begin{aligned}
\varepsilon_{1}(t) \Lambda f_{1} & \leq \Lambda\left|\varepsilon_{1}(t)\right|\left|x_{1}\right| \Upsilon_{1} \leq \Lambda\left|\varepsilon_{1}(t)\right| \Upsilon_{1}\left(\rho_{1}(t)\left|\varepsilon_{1}(t)\right|+\left|\gamma_{d}\right|\right) \\
& \leq \Lambda\left[\varepsilon_{1}(t)\right]^{2} \Upsilon_{1} \rho_{1}(t)+\left(\Lambda \Upsilon_{1}\left|\varepsilon_{1}(t)\right|\left|\gamma_{d}\right|\right)^{2}+\frac{1}{4} .
\end{aligned}
$$

Choose the following Lyapunov function:

$$
L_{1}=\frac{1}{2}\left[\varepsilon_{1}(t)\right]^{2}
$$


The time derivative of (25) is as follows:

$$
\begin{aligned}
L_{1} \leq & \varepsilon_{1}(t)\left[\Gamma+\Lambda\left(f_{1}+e_{2}+\alpha_{1}-\dot{\gamma}_{d}\right)\right] \\
\leq & \varepsilon_{1}(t)\left[\Gamma+\Lambda\left(e_{2}+\alpha_{1}-\dot{\gamma}_{d}\right)\right]+\varepsilon_{1}(t) \Lambda f_{1} \\
\leq & \varepsilon_{1}(t)\left[\Gamma+\Lambda\left(e_{2}+\alpha_{1}-\dot{\gamma}_{d}\right)\right]+\left[\varepsilon_{1}(t)\right]^{2} \rho_{1}(t) \Lambda \Upsilon_{1} \\
& +\left(\Lambda \Upsilon_{1}\left|\varepsilon_{1}(t)\right|\left|\gamma_{d}\right|\right)^{2}+\frac{1}{4} \\
& \quad \\
= & \varepsilon_{1}(t)\left[\Gamma+\Lambda\left(e_{2}+\alpha_{1}-\dot{\gamma}_{d}+\Upsilon_{1} \rho_{1}(t) \varepsilon_{1}(t)+\Lambda \Upsilon_{1}^{2} \gamma_{d}^{2} \varepsilon_{1}(t)\right)\right]+\frac{1}{4} .
\end{aligned}
$$

Thus, choose the virtual control law as

$$
\alpha_{1}=\frac{1}{\Lambda}\left[-k_{1} \varepsilon_{1}(t)-\Gamma-\Lambda\left(\Upsilon_{1} \rho_{1}(t) \varepsilon_{1}(t)+\Lambda \Upsilon_{1}^{2} \gamma_{d}^{2} \varepsilon_{1}(t)-\dot{\gamma}_{d}\right)\right]
$$

where $k_{1}$ is the design parameter. Substituting (27) into (26) results in

$$
\dot{L}_{1} \leq-k_{1}\left[\varepsilon_{1}(t)\right]^{2}+\Lambda \varepsilon_{1}(t) e_{2}
$$

Step 2. Similar to Step 1, there is

$$
\begin{aligned}
e_{2} f_{2} & =\left|e_{2}\right| \Upsilon_{2}\left(\left|x_{1}\right|+\left|x_{2}\right|\right) \\
& \leq\left|e_{2}\right| \Upsilon_{2}\left(\rho_{1}(t)\left|\varepsilon_{1}(t)\right|+\left|\gamma_{d}\right|+\left|e_{2}\right|+\left|\alpha_{1}\right|\right) \\
& =\Upsilon_{2} e_{2}^{2}+\left|e_{2}\right| \Upsilon_{2}\left(\rho_{1}(t)\left|\varepsilon_{1}(t)\right|+\left|\gamma_{d}\right|+\left|\alpha_{1}\right|\right) \\
& \leq \Upsilon_{2} e_{2}^{2}+\left[\left|e_{2}\right| \Upsilon_{2}\left(\rho_{1}(t)\left|\varepsilon_{1}(t)\right|+\left|\gamma_{d}\right|+\left|\alpha_{1}\right|\right)\right]^{2}+\frac{1}{4}
\end{aligned}
$$

Choose the following Lyapunov function:

$$
L_{2}=L_{1}+\frac{1}{2} e_{2}^{2}
$$

The time derivative of (30) is as follows:

$$
\begin{aligned}
\dot{L}_{2}= & \dot{L}_{1}+e_{2}\left(f_{2}+x_{3}-\dot{\alpha}_{1}\right) \\
\leq & -k_{1}\left[\varepsilon_{1}(t)\right]^{2}+\varepsilon_{1}(t) \Lambda e_{2}+\frac{1}{4}+e_{2}\left(e_{3}+x_{3}-\dot{\alpha}_{1}\right) \\
& +\gamma_{2} e_{2}^{2}+e_{2}^{2}\left[\Upsilon_{2}\left(\rho_{1}(t)\left|\varepsilon_{1}(t)\right|+\left|\gamma_{d}\right|+\left|\alpha_{1}\right|\right)\right]^{2} \\
& +\frac{1}{4}=-k_{1}\left[\varepsilon_{1}(t)\right]^{2}+\frac{1}{2}+e_{2}\left[\varepsilon_{1}(t) \Lambda+e_{3}+\alpha_{2}-\dot{\alpha}_{1}\right. \\
& \left.+r_{2} e_{2}+e_{2}\left(\Upsilon_{2}\left(\rho_{1}(t)\left|\varepsilon_{1}(t)\right|+\left|\gamma_{d}\right|+\left|\alpha_{1}\right|\right)\right)^{2}\right] .
\end{aligned}
$$

Thus, choose the virtual control law as

$$
\begin{aligned}
\alpha_{2}= & -k_{2} e_{2}-\varepsilon_{1}(t) \Lambda-\Upsilon_{2} e_{2} \\
& -e_{2}\left(\Upsilon_{2}\left(\rho_{1}(t)\left|\varepsilon_{1}(t)\right|+\left|\gamma_{d}\right|+\left|\alpha_{1}\right|\right)\right)^{2}+\dot{\alpha}_{1},
\end{aligned}
$$

TABLe 1: HFV state initial value.

\begin{tabular}{lcc}
\hline Item & Value & Units \\
\hline$V$ & 2500 & $\mathrm{~m} / \mathrm{s}$ \\
$h$ & 27000 & $\mathrm{~m}$ \\
$\gamma$ & 0 & $\circ$ \\
$\theta$ & 1.5295 & $\circ$ \\
$Q$ & 0 & $\%$ \\
$\eta_{1}$ & 0.2857 & - \\
$\eta_{2}$ & 0.2350 & - \\
\hline
\end{tabular}

where $k_{2}$ is the design parameter. Substituting (32) into (31) results in

$$
\dot{L}_{2} \leq-k_{1}\left[\varepsilon_{1}(t)\right]^{2}-k_{2} e_{2}^{2}+e_{2} e_{3}+\frac{1}{2}
$$

Step 3. Similar to Step 2, there is

$e_{3} f_{3} \leq Y_{3} e_{3}^{2}+\left[\left|e_{3}\right| Y_{3}\left(\rho_{1}(t)\left|\varepsilon_{1}(t)\right|+\left|\gamma_{d}\right|+\left|e_{2}\right|+\left|\alpha_{1}\right|+\left|\alpha_{2}\right|\right)\right]^{2}+\frac{1}{4}$

Choose the following Lyapunov function:

$$
L_{3}=L_{2}+\frac{1}{2} e_{3}^{2}
$$

The time derivative of (35) is as follows:

$$
\begin{aligned}
\dot{L}_{3}= & \dot{L}_{2}+e_{3}\left(f_{3}+\delta_{e}-\dot{\alpha}_{2}\right) \\
\leq & -k_{1}\left[\varepsilon_{1}(t)\right]^{2}-k_{2} e_{2}^{2}-k_{3} e_{3}^{2}+\frac{3}{4} \\
& +e_{3}\left\{e_{3}\left[\gamma_{3}\left(\rho_{1}(t)\left|\varepsilon_{1}(t)\right|+\left|\gamma_{d}\right|+\left|e_{2}\right|+\left|\alpha_{1}\right|+\left|\alpha_{2}\right|\right)\right]^{2}\right. \\
& \left.+e_{2}+\gamma_{3} e_{3}+\delta_{e}-\dot{\alpha}_{2}\right\} .
\end{aligned}
$$

Thus, choose the control law as

$$
\begin{aligned}
\delta_{e}= & -k_{3} e_{3}-e_{2}-\gamma_{3} e_{3}-e_{3}\left[\gamma _ { 3 } \left(\rho_{1}(t)\left|\varepsilon_{1}(t)\right|+\left|\gamma_{d}\right|\right.\right. \\
& \left.\left.+\left|e_{2}\right|+\left|\alpha_{1}\right|+\left|\alpha_{2}\right|\right)\right]^{2}+\dot{\alpha}_{2}
\end{aligned}
$$

where $k_{3}$ is the design parameter. It follows from (37) and (36) that (38) holds

$$
\dot{L}_{3} \leq-k_{1}\left[\varepsilon_{1}(t)\right]^{2}-k_{2} e_{2}^{2}+k_{3} e_{3}^{2}+\frac{3}{4}=-k L_{3}+\frac{3}{4},
$$

where $k=\min \left\{2 k_{1}, 2 k_{2}, 2 k_{3}\right\}$. Then, solving the above differential equation, there is

$$
L_{3} \leq e^{-k t}\left[L_{3}(0)-\frac{3}{4}\right]+\frac{3}{4} .
$$



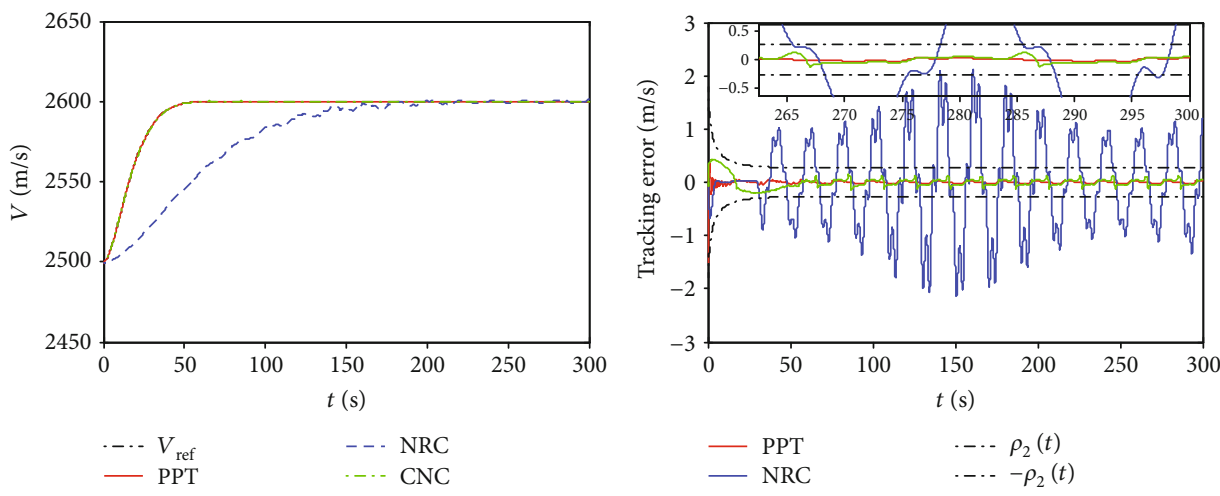

(a) Velocity tracking performance
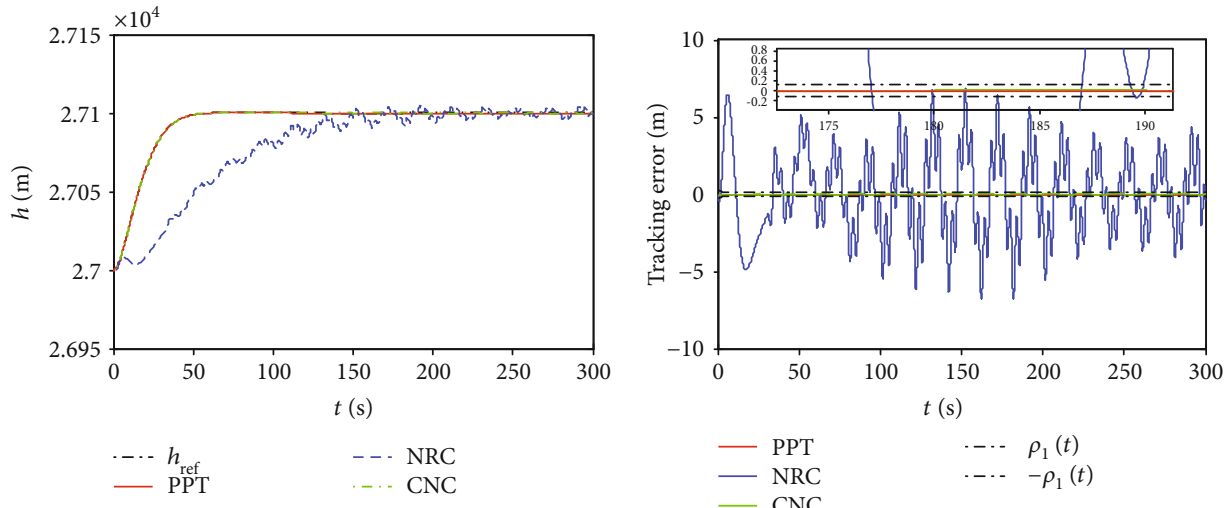

$$
\begin{array}{lll}
\text { PPT } & \cdots & \rho_{1}(t) \\
\text { - NRC } & \cdots & -\rho_{1}(t) \\
\text { CNC } & &
\end{array}
$$

(b) Altitude tracking performance
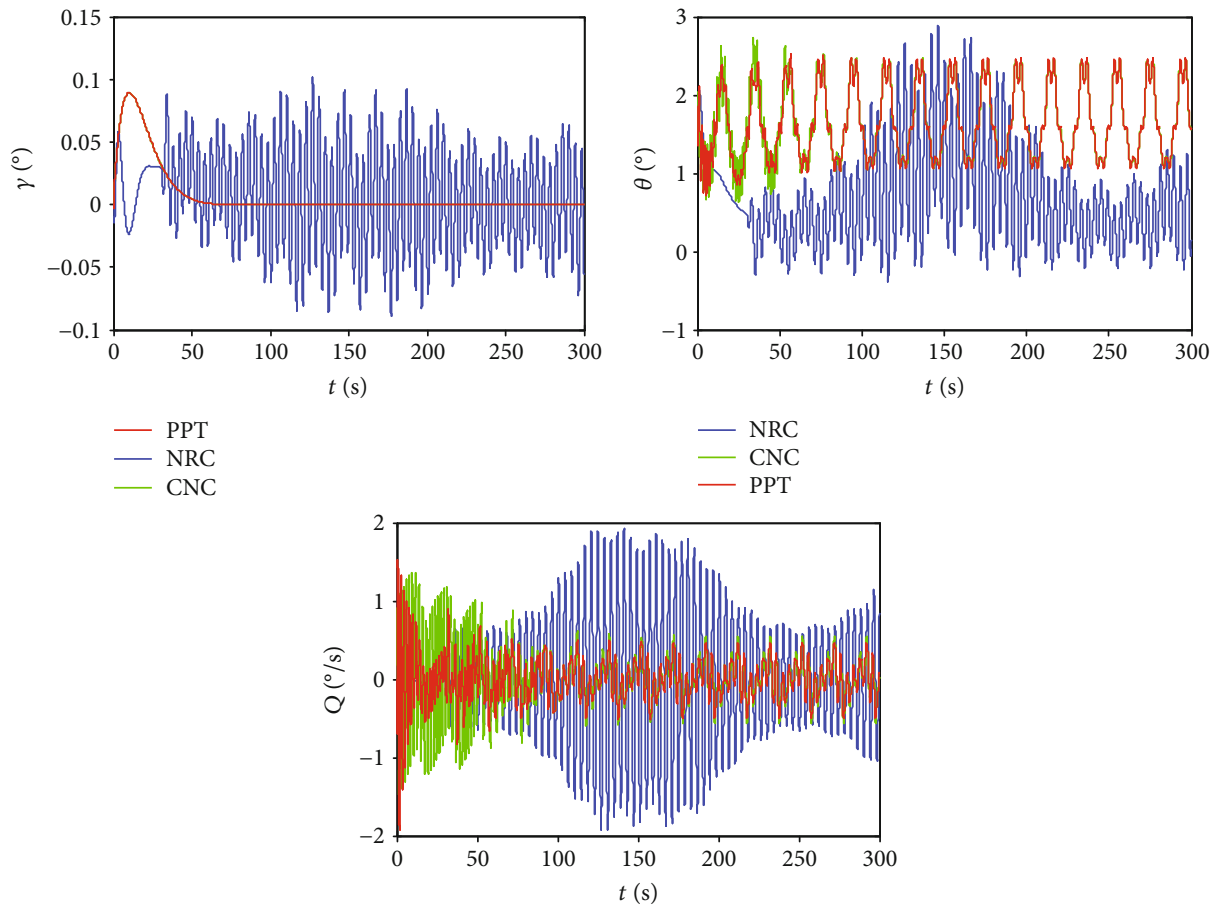

- NRC

(c) Altitude angle

Figure 2: Continued. 

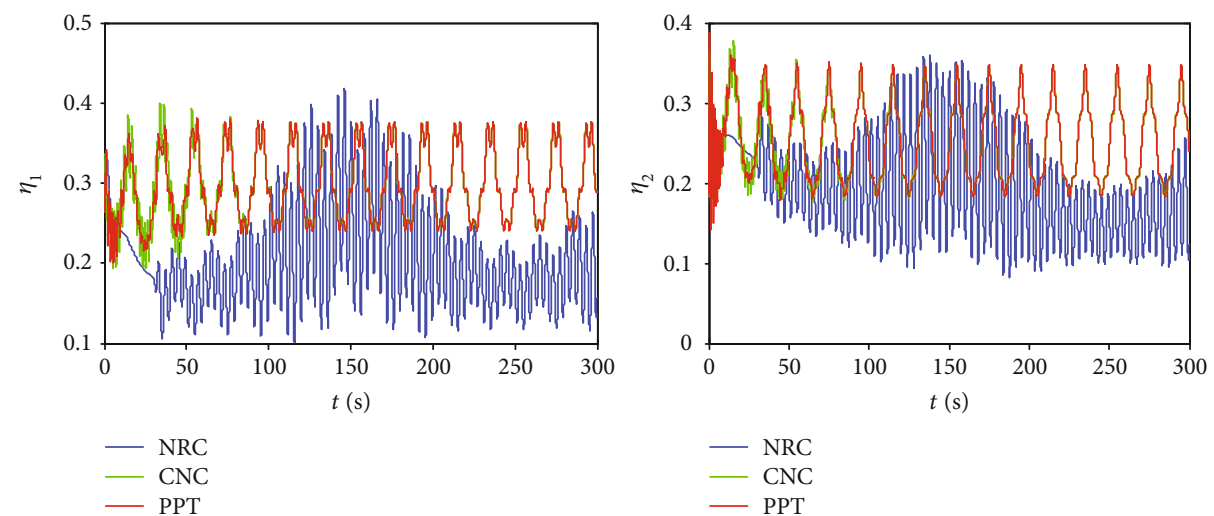

(d) Elastic states
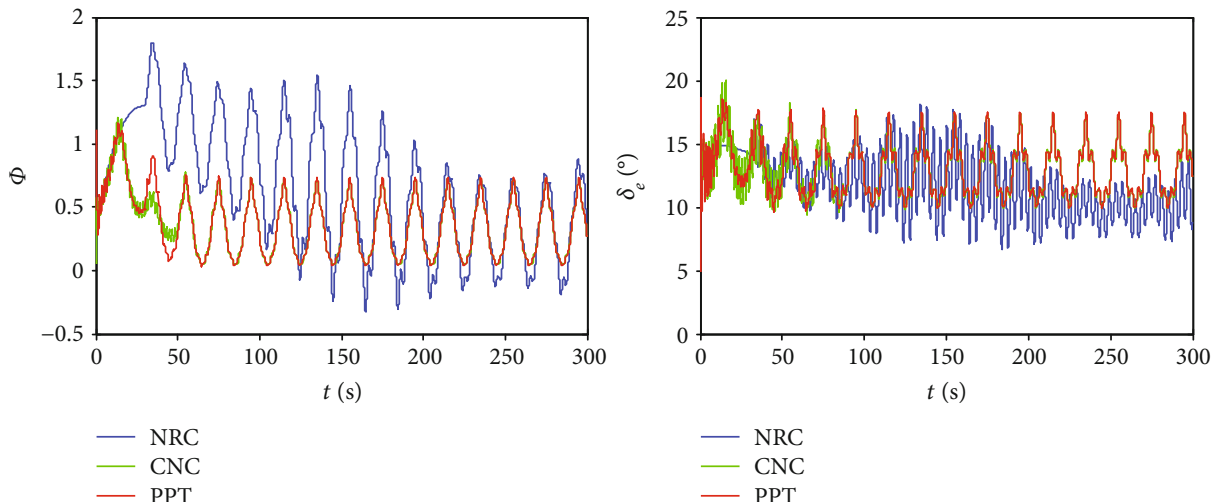

- NRC

- CNC

(e) Control inputs
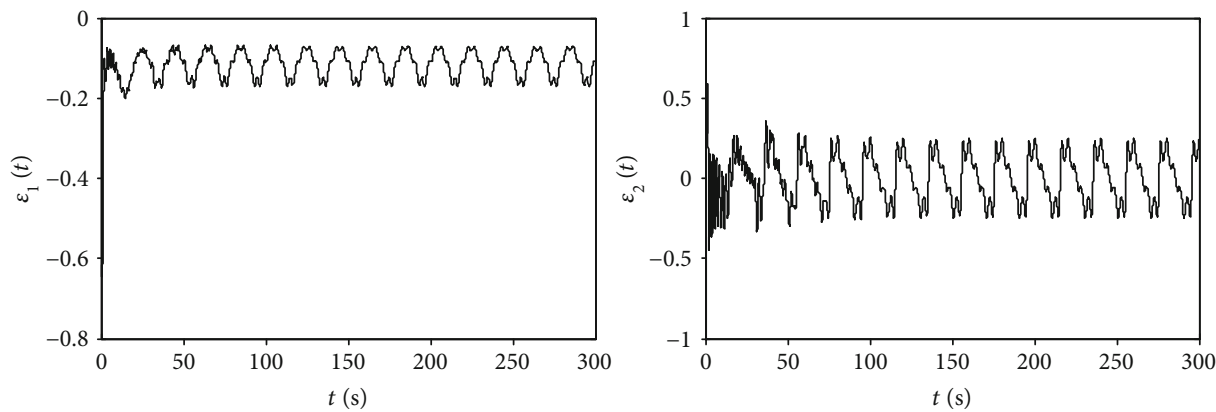

(f) Transform error

Figure 2: Case 1 simulation diagrams.

Obviously, from Lemma 2, transform error $\varepsilon_{1}(t)$ and tracking errors $e_{2}$ and $e_{3}$ are bounded. Furthermore, all the signals in the closed-loop are bounded. Thus, the transient performance and steady-state accuracy of the altitude subsystem are guaranteed.

3.2. Velocity Control Design. A PI controller is designed for the velocity subsystem because the velocity dynamics is much simpler than the altitude dynamic change.

$$
\Phi=-k_{V 1} \varepsilon_{2}(t)-k_{V 2} \int_{0}^{t} \varepsilon_{2}(t) d t+\Phi_{0}
$$

with $\varepsilon_{2}(t)=\ln \left(\left(\tilde{V} / \rho_{2}(t)+1\right) /\left(1-\tilde{V} / \rho_{2}(t)\right)\right), \quad \tilde{V}=V-V_{\text {ref }}$, and $\rho_{2}(t)=\operatorname{csch}\left(l_{2} t+\rho_{20}\right)+\rho_{2 \infty}$. The correction term $\Phi_{0}$ is introduced into the control law so that the tracking error can converge to zero.

Since the stability of the PI controller has been proven many times in early research, it will not be repeated here. Obviously, the boundedness of $\varepsilon_{2}(t)$ can be guaranteed via the PI control law. Furthermore, all the signals in the closed-loop are bounded. Thus, the prescribed transient performance and steady-state accuracy of the velocity subsystem are achieved.

\section{Simulation Results}

In order to verify the practicability of the designed controllers, this section will prove this via digital simulation 

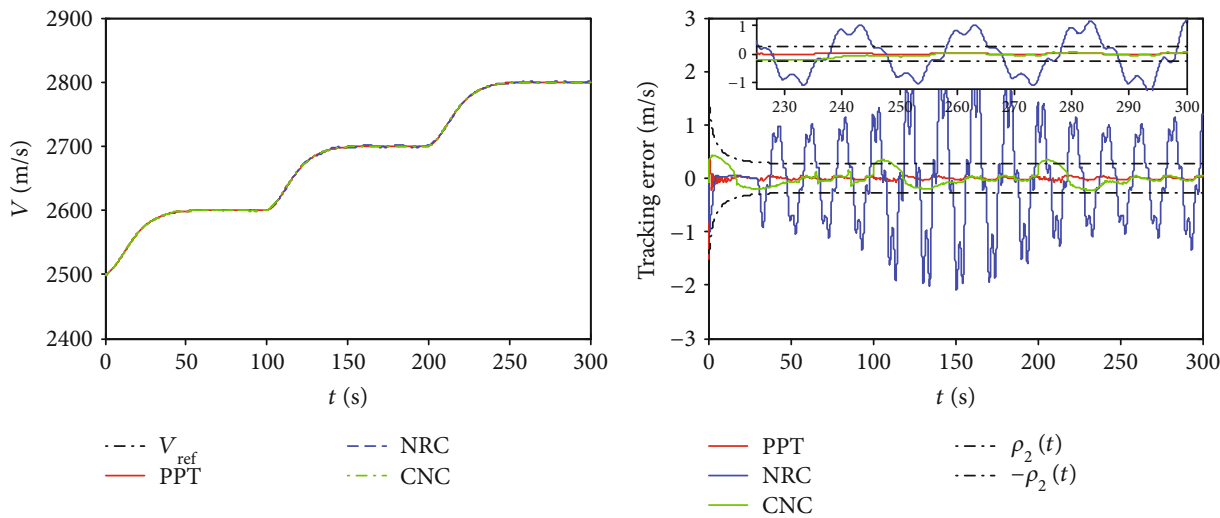

(a) Velocity tracking performance
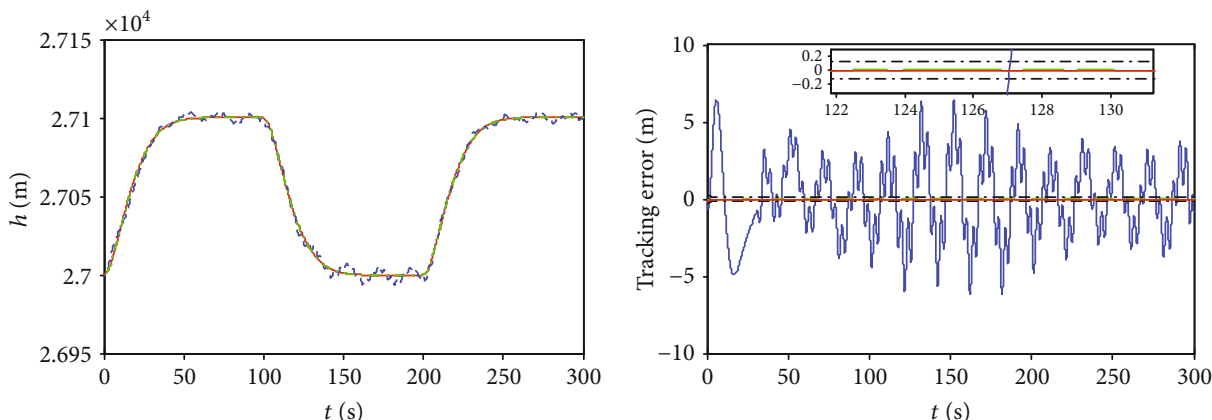

$\begin{array}{ll}\cdots & h_{\text {ref }} \\ - & \text { PPT }\end{array}$

$-\cdots \mathrm{NRC}$
$-\cdots \mathrm{CNC}$

— PPT

$\cdots \rho_{1}(t)$

- CNC

(b) Altitude tracking performance
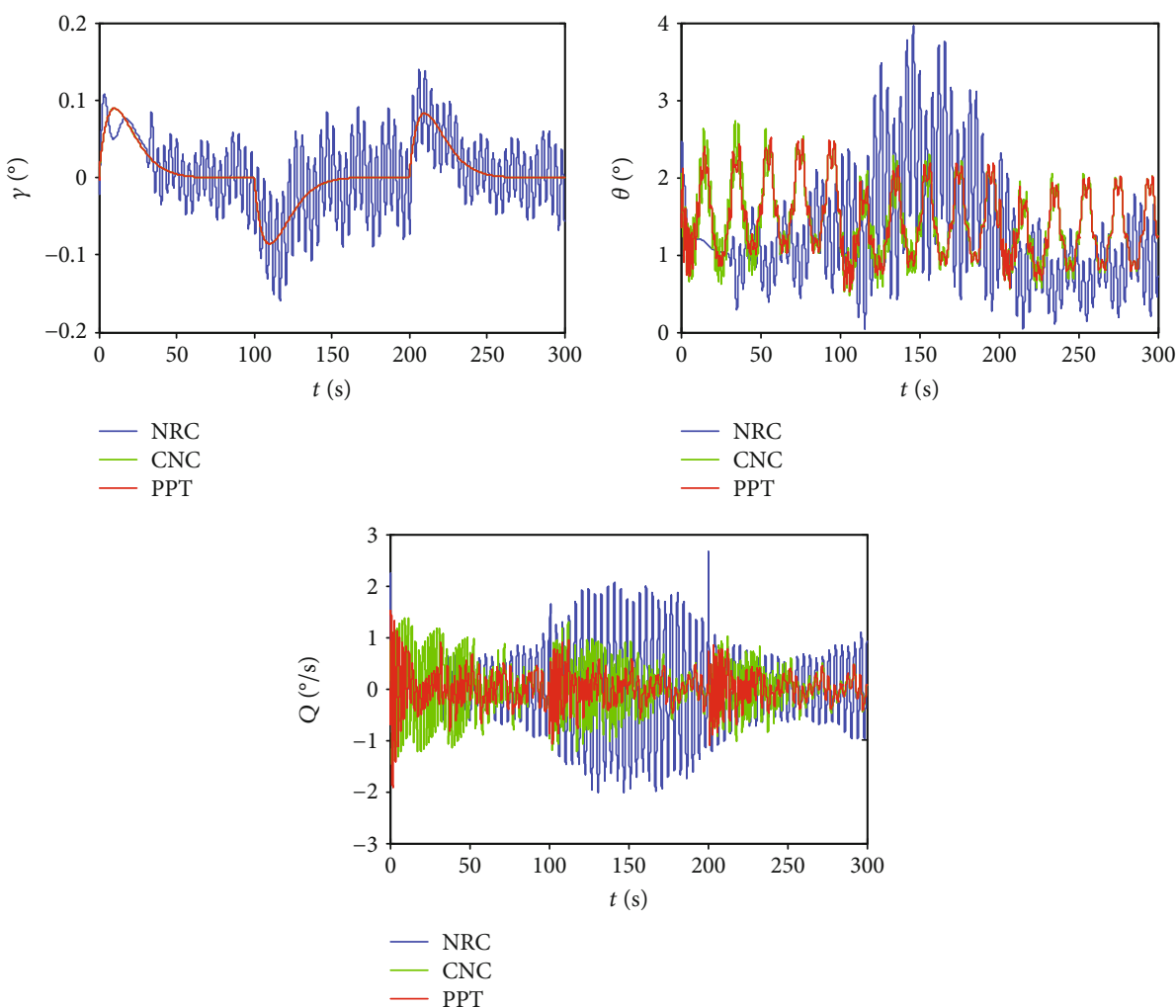

(c) Altitude angle

Figure 3: Continued. 

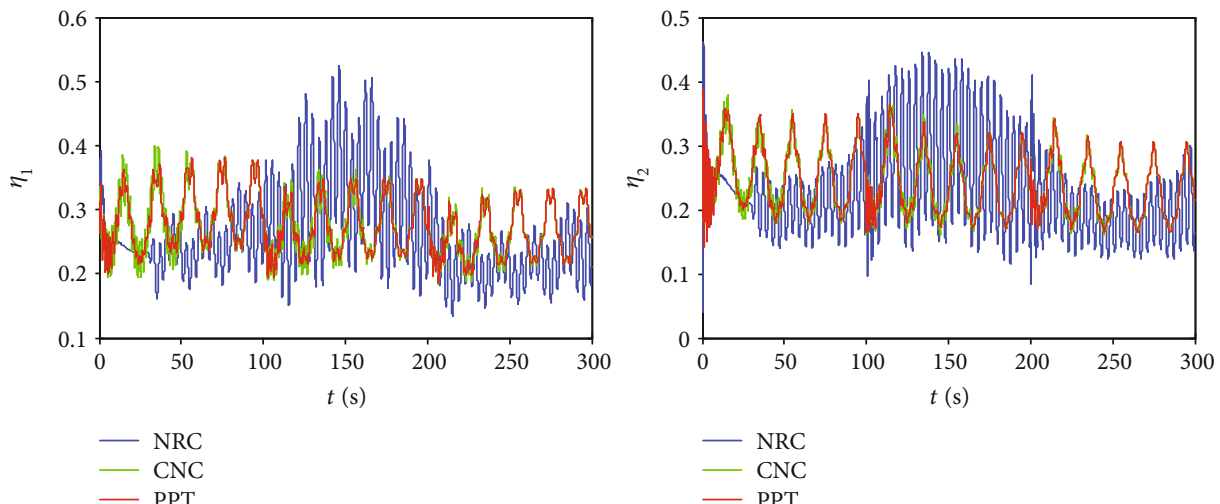

(d) Elastic states
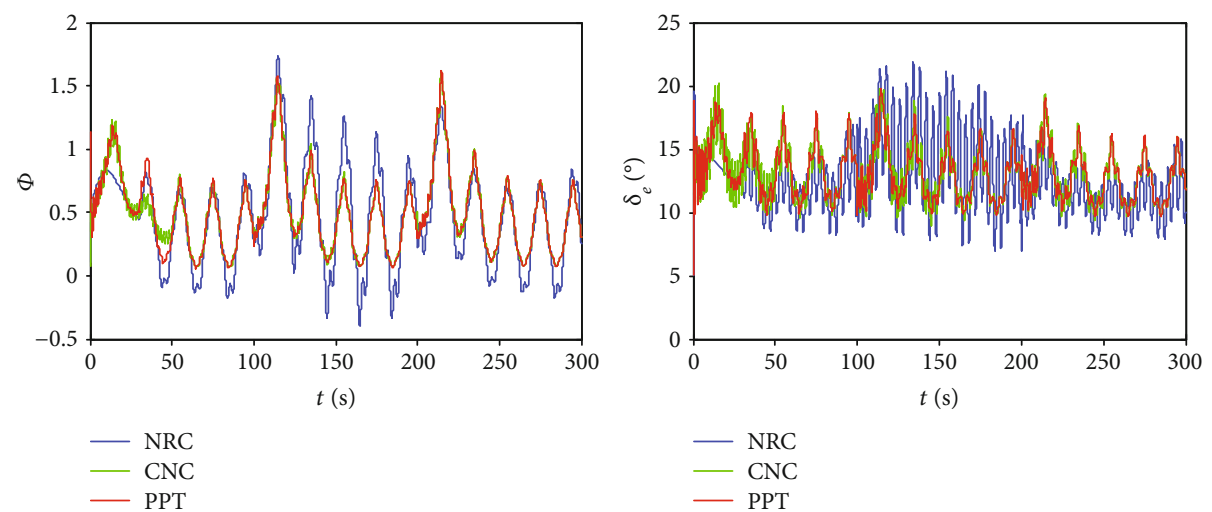

(e) Control inputs
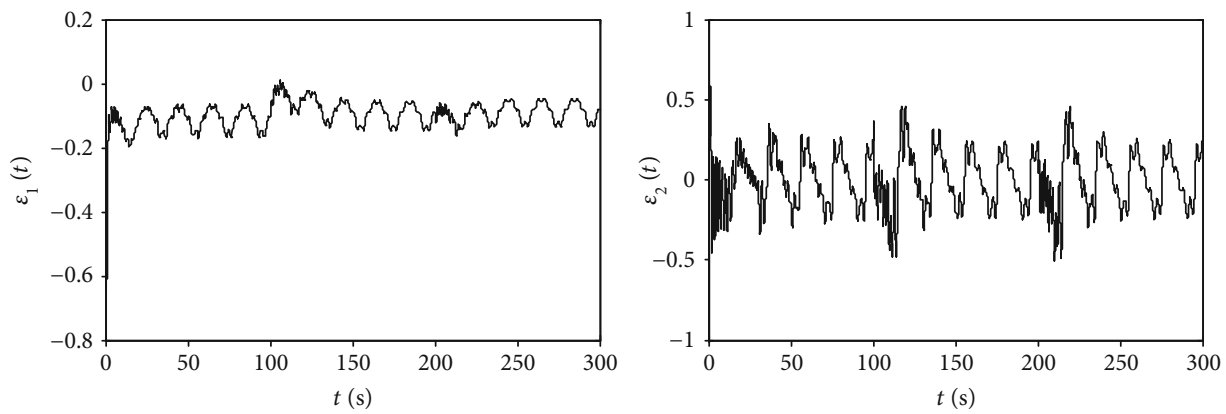

(f) Transform error

FIgURE 3: Case 2 simulation diagrams.

experiments. The performance functions are selected as $\rho_{h}(t)=\operatorname{csch}(0.1 t+0.3)+1.8, \quad \rho_{1}(t)=\operatorname{csch}(0.15 t+2.8)+$ 0.05 , and $\rho_{2}(t)=\operatorname{csch}(0.1 t+0.2)+0.9$. The design parameters are chosen as follows: $k_{h}=8, k_{1}=0.05, k_{2}=0.1, k_{3}=0.8$, $k_{V 1}=0.6, k_{V 2}=1$, and $\Phi_{0}=0.38$. Meanwhile, the pneumatic parameters to account for uncertainties in the HFV dynamics are changed by defining $C=C_{0}[1+0.3 \sin (0.1 \pi t)]$. The initial trim conditions of HFV are listed in Table 1. In the simulation process, when the time exceeds $30 \mathrm{~s}$, the velocity subsystem and the altitude subsystem are interfered with $d_{V}=5 \sin (0.1 \pi t)$ and $d_{h}=0.02 \sin (0.5 \pi t)$, respectively. In order to show the superiority of the proposed controller, the simulation results compare the prescribed performance tracking controller (PPT) with the novel robust control
(NRC) in Ref. [39] and the concise neural control (CNC) in Ref. [40]. At the same time, the simulation experiments are carried out in the following two cases:

Case 1. The HFV velocity step and altitude step of cruise flight are required to be $100 \mathrm{~m} / \mathrm{s}$ and $100 \mathrm{~m}$, respectively.

Case 2. The HFV velocity input for cruise flight is required to be a signal with a step amplitude of $100 \mathrm{~m} / \mathrm{s}$ per 100 seconds, and the height input is a square wave signal with an amplitude of $100 \mathrm{~m}$ and a period of 200 seconds.

The simulation results are shown in Figures 2 and 3. From the velocity and altitude tracking performance, three methods can realize stable tracking of reference inputs 
under the premise of interference. The PPT method has smaller overshoot and faster convergence speed. The altitude angle and elastic states of the three methods do not appear as high-frequency chattering phenomenon, but the PPT method has a smoother response. The control inputs of the three methods can be stable within the limited range, and the PPT method also has a smoother response. The transform error functions in both cases are bounded.

\section{Conclusions}

In this paper, a prescribed performance tracking control is designed for HFV with model uncertainties. Based on the timescale principle, the HFV longitudinal motion model is decomposed into the velocity subsystem and the altitude subsystem. A novel performance function is designed which does not depend on the initial error. The performance function limits the tracking error within the prescribed range so that the system has satisfactory transient performance and steady-state accuracy. The control objective of the altitude subsystem is changed by model transformation, and the prescribed performance backstepping controller is designed. The prescribed performance PI controller is proposed for the velocity subsystem which has better engineering practicability. Obviously, the designed controllers are more convenient. Finally, the simulation results show that the proposed controller has good practicability. Meanwhile, compared with two other control methods, it has better transient performance and steadystate accuracy and a smoother response.

\section{Data Availability}

The data used to support the findings of this study are included within the supplementary information file.

\section{Conflicts of Interest}

The authors declare that there is no conflict of interest regarding the publication of this paper.

\section{Acknowledgments}

This work was supported by the National Natural Science Foundation of China (Grant No. 61603410).

\section{Supplementary Materials}

The model parameters and aerodynamic coefficient data of HFV used to support the findings of this study are included within the supplementary information file. (Supplementary Materials)

\section{References}

[1] L. F. Peter, "USAF to develop near-space UAVs," Flight International, vol. 172, no. 5102, p. 10, 2007.

[2] T. V. Markova, A. A. Aksenov, S. V. Zhluktov, D. V. Savitskiy, A. D. Gavrilov, and E. E. Son, "Numerical simulation of gas flow past scale model of hypersonic vehicle in wind tunnel," High Temperature, vol. 55, no. 2, pp. 280-285, 2017.

[3] W. Zhu, J. Li, and Y. Xu, "Optimum attitude planning of nearspace solar powered airship," Aerospace Science and Technology, vol. 84, pp. 291-305, 2019.

[4] L. Fiorentini, A. Serrani, M. A. Bolender, and D. B. Doman, "Nonlinear robust adaptive control of flexible air-breathing hypersonic vehicles," Journal of Guidance, Control, and Dynamics, vol. 32, no. 2, pp. 402-417, 2009.

[5] X. Bu, Y. Xiao, and H. Lei, "An adaptive critic design-based fuzzy neural controller for hypersonic vehicles: predefined behavioral nonaffine control," IEEE/ASME Transactions on Mechatronics, vol. 24, no. 4, pp. 1871-1881, 2019.

[6] C. Mu, Z. Ni, C. Sun, and H. He, "Air-breathing hypersonic vehicle tracking control based on adaptive dynamic programming," IEEE Transactions on Neural Networks and Learning Systems, vol. 28, no. 3, pp. 584-598, 2017.

[7] J. T. Parker, A. Serrani, S. Yurkovich, M. A. Bolender, and D. B. Doman, "Control-oriented modeling of an air-breathing hypersonic vehicle," Journal of Guidance, Control, and Dynamics, vol. 30, no. 3, pp. 856-869, 2007.

[8] S. Zhao and X. Li, "Prescribed performance fault tolerant control for hypersonic flight vehicles with actuator failures," IEEE Access, vol. 7, pp. 100187-1100204, 2019.

[9] X. Bu, H. Lei, and Y. Gao, "Robust tracking control of hypersonic flight vehicles: a continuous model-free control approach," Acta Astronautica, vol. 161, pp. 234-240, 2019.

[10] P. Wang, G. J. Tang, and J. Wu, "Sliding mode decoupling control of a generic hypersonic vehicle based on parametric commands," Science China Information Sciences, vol. 58, no. 5, pp. 1-14, 2015.

[11] J. G. Sun and S. M. Song, "Tracking control of hypersonic vehicles with input saturation based on fast terminal sliding mode," International Journal of Aeronautical and Space Sciences, vol. 20, no. 2, pp. 493-505, 2019.

[12] B. Jiang, P. Shi, C. C. Lim, and D. Xu, "Adaptive neural observer-based backstepping fault tolerant control for near space vehicle under control effector damage," IET Control Theory \& Applications, vol. 8, no. 9, pp. 658-666, 2014.

[13] S. Zhang, Q. Wang, G. Yang, and M. Zhang, "Anti-disturbance backstepping control for air-breathing hypersonic vehicles based on extended state observer," ISA Transactions, 2019.

[14] Z. Zhao, J. Yu, L. Zhao, H. Yu, and C. Lin, “Adaptive fuzzy control for induction motors stochastic nonlinear systems with input saturation based on command filtering," Information Sciences, vol. 463-464, pp. 186-195, 2018.

[15] J. Yu, P. Shi, C. Lin, and H. Yu, "Adaptive neural command filtering control for nonlinear MIMO systems with saturation input and unknown control direction," IEEE Transactions on Cybernetics, pp. 1-10, 2019.

[16] J. Yu, B. Chen, H. Yu, C. Lin, and L. Zhao, "Neural networksbased command filtering control of nonlinear systems with uncertain disturbance," Information Sciences, vol. 426, pp. 50-60, 2018.

[17] P. Wang, J. Wang, X. Bu, C. Luo, and S. Tan, “Adaptive fuzzy back-stepping control of a flexible air-breathing hypersonic vehicle subject to input constraints," Journal of Intelligent \& Robotic Systems, vol. 87, no. 3-4, pp. 565-582, 2017.

[18] Q. Hu and Y. Meng, "Adaptive backstepping control for airbreathing hypersonic vehicle with actuator dynamics," Aerospace Science and Technology, vol. 67, pp. 412-421, 2017. 
[19] Q. Hu, Y. Meng, C. Wang, and Y. Zhang, “Adaptive backstepping control for air-breathing hypersonic vehicles with input nonlinearities," Aerospace Science and Technology, vol. 73, pp. 289-299, 2018.

[20] X. Bu and Y. Xiao, "Prescribed performance-based lowcomputational cost fuzzy control of a hypersonic vehicle using non-affine models," Advances in Mechanical Engineering, vol. 10, no. 2, 2018.

[21] X. Bu, X. Wu, J. Huang, and D. Wei, "Robust estimationfree prescribed performance back-stepping control of airbreathing hypersonic vehicles without affine models," International Journal of Control, vol. 89, no. 11, pp. 2185-2200, 2016.

[22] C. P. Bechlioulis and G. A. Rovithakis, "Robust adaptive control of feedback linearizable MIMO nonlinear systems with prescribed performance," IEEE Transactions on Automatic Control, vol. 53, no. 9, pp. 2090-2099, 2008.

[23] Y. Wang and J. Hu, "Improved prescribed performance control for air-breathing hypersonic vehicles with unknown deadzone input nonlinearity," ISA Transactions, vol. 79, pp. 95-107, 2018.

[24] C. Mu and Y. Zhang, "Learning-based robust tracking control of quadrotor with time-varying and coupling uncertainties," IEEE Transactions on Neural Networks and Learning Systems, pp. 1-15, 2019.

[25] J. D. Shaughnessy, S. Z. Pinckney, J. D. McMinn, C. I. Cruz, and M. L. Kelley, "Hypersonic vehicle simulation model: winged-cone configuration," Tech. Rep. NASA TM-102610, NASA Langley Research Center, 1990.

[26] F. R. Chavez and D. K. Schmidt, "Analytical aeropropulsiveaeroelastic hypersonic-vehicle model with dynamic analysis," Journal of Guidance, Control, and Dynamics, vol. 17, no. 6, pp. 1308-1319, 1994.

[27] M. A. Bolender, "An overview on dynamics and controls modelling of hypersonic vehicles," in 2009 American Control Conference, pp. 2507-2512, St. Louis, MO, USA, 2009.

[28] M. A. Bolender and D. B. Doman, "Nonlinear longitudinal dynamical model of an air-breathing hypersonic vehicle," Journal of Spacecraft and Rockets, vol. 44, no. 2, pp. 374387, 2007.

[29] C. P. Bechlioulis and G. A. Rovithakis, "Prescribed performance adaptive control for multi-input multi-output affine in the control nonlinear systems," IEEE Transactions on Automatic Control, vol. 55, no. 5, pp. 1220-1226, 2010.

[30] C. P. Bechlioulis and G. A. Rovithakis, "Robust partial-state feedback prescribed performance control of cascade systems with unknown nonlinearities," IEEE Transactions on Automatic Control, vol. 56, no. 9, pp. 2224-2230, 2011.

[31] B. Xu, C. Yang, and Y. Pan, "Global neural dynamic surface tracking control of strict-feedback systems with application to hypersonic flight vehicle," IEEE Transactions on Neural Networks and Learning Systems, vol. 26, no. 10, pp. 25632575, 2015.

[32] X. Bu, X. Wu, J. Huang, and D. Wei, “A guaranteed transient performance-based adaptive neural control scheme with lowcomplexity computation for flexible air-breathing hypersonic vehicles," Nonlinear Dynamics, vol. 84, no. 4, pp. 2175-2194, 2016.

[33] B. Xu, "Robust adaptive neural control of flexible hypersonic flight vehicle with dead-zone input nonlinearity," Nonlinear Dynamics, vol. 80, no. 3, pp. 1509-1520, 2015.
[34] X. Huang, W. Lin, and B. Yang, "Global finite-time stabilization of a class of uncertain nonlinear systems," Automatica, vol. 41, no. 5, pp. 881-888, 2005.

[35] Z. Zhang, G. Duan, and M. Hou, "Global finite time stabilization of pure-feedback systems with input dead-zone nonlinearity," Journal of the Franklin Institute, vol. 354, no. 10, pp. 4073-4101, 2017.

[36] S. I. Han and J. M. Lee, "Fuzzy echo state neural networks and funnel dynamic surface control for prescribed performance of a nonlinear dynamic system," IEEE Transactions on Industrial Electronics, vol. 61, no. 2, pp. 1099-1112, 2014.

[37] J. Wu, W. Chen, and J. Li, "Global finite-time adaptive stabilization for nonlinear systems with multiple unknown control directions," Automatica, vol. 69, pp. 298-307, 2016.

[38] J. Li, J. Wu, X. Guo, X. Li, and L. Ai, "Global finite-time stabilization for a class of high-order nonlinear systems with multiple unknown control directions," International Journal of Control, Automation and Systems, vol. 15, no. 1, pp. 178185, 2017.

[39] W. Tang, W. Long, and H. Gao, "A novel robust flight controller design for an air-breathing hypersonic vehicle," Automatic Control and Computer Sciences, vol. 52, no. 3, pp. 198-207, 2018.

[40] X. Bu, Q. Wang, Y. Zhao, and G. He, "Concise neural nonaffine control of air-breathing hypersonic vehicles subject to parametric uncertainties," International Journal of Aerospace Engineering, vol. 2017, Article ID 1374932, 16 pages, 2017. 


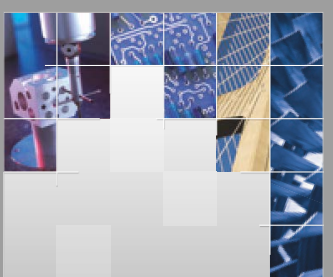

\section{Enfincering}
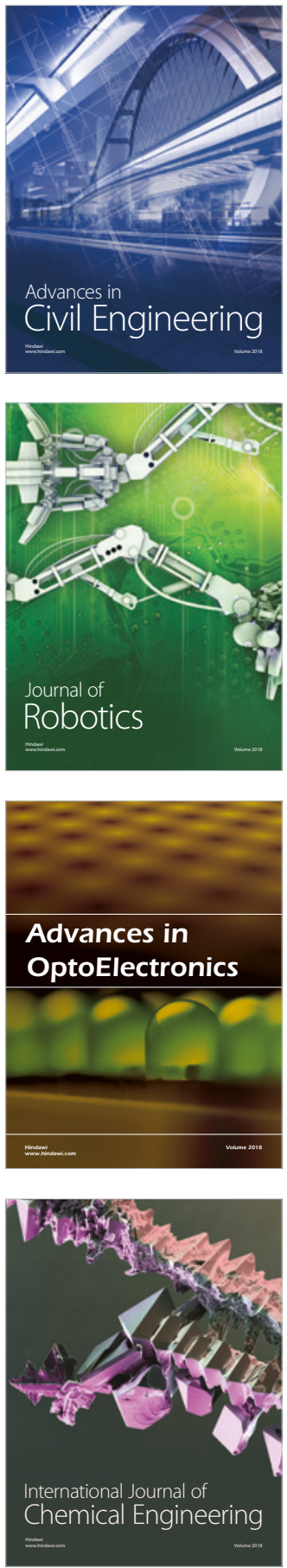

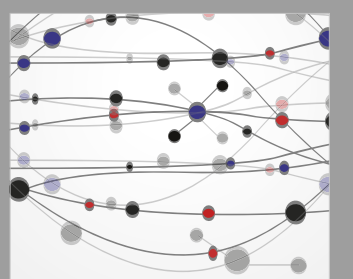

\section{Rotating \\ Machinery}

The Scientific World Journal

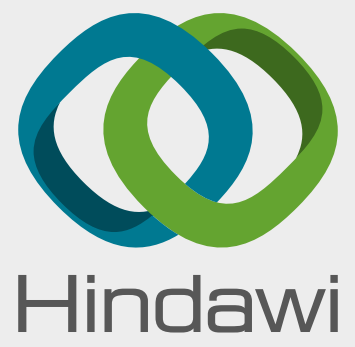

Submit your manuscripts at

www.hindawi.com
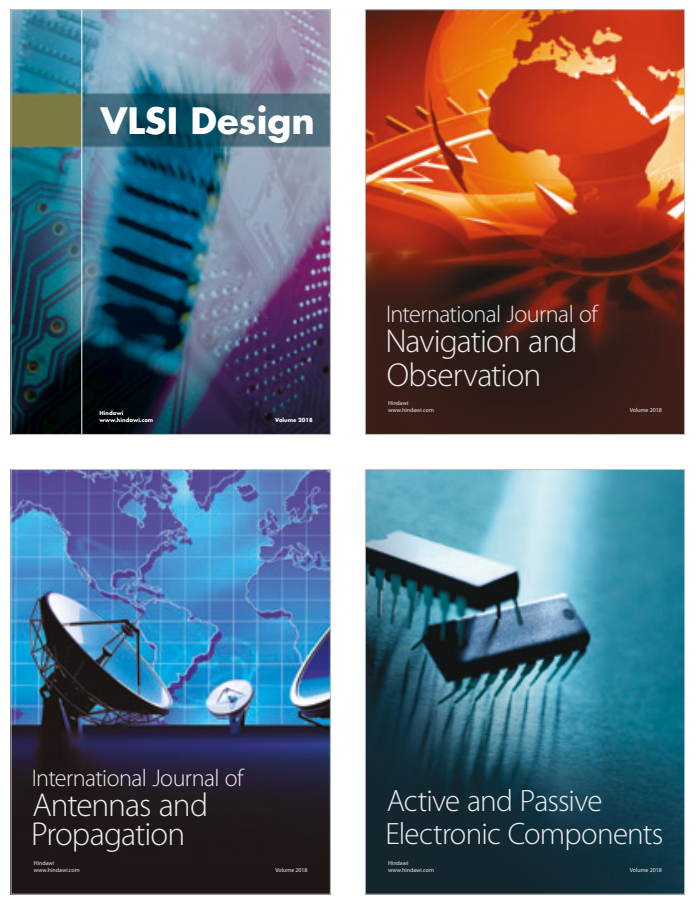
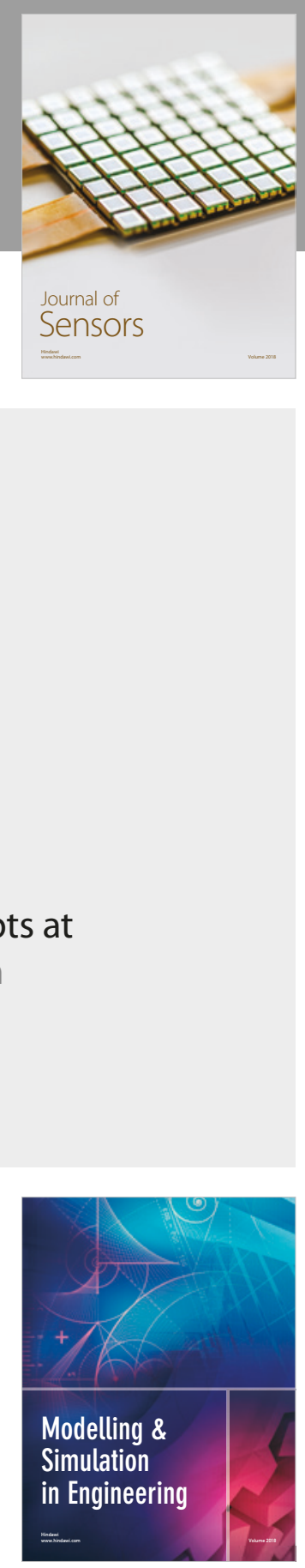

\section{Advances \\ Multimedia}
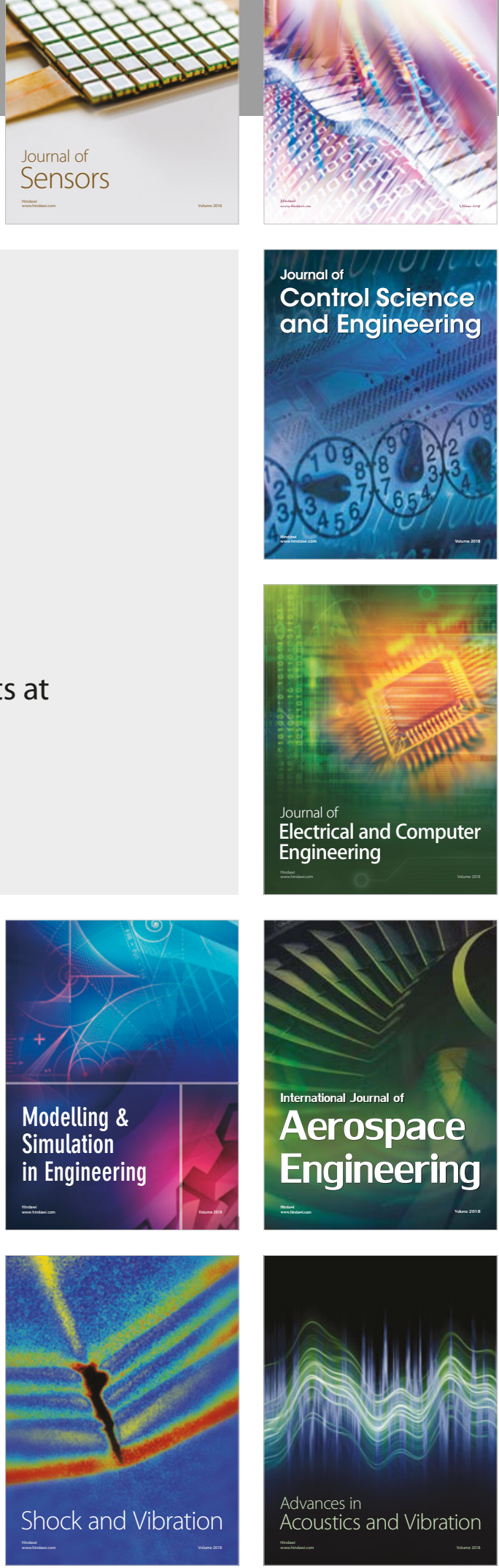